\title{
Clinical - Radiological Correlates in Intracerebral Hematomas due to Aneurysmal Rupture
}

\author{
B.G. BENOIT, D.D. COCHRANE, F. DURITY, G.G. FERGUSON, D. FEWER, K.M. HUNTER, M.I. KHAN, G. MOHR,
} A.R. WATTS, B.K.A. WEIR, W.B. WHEELOCK

SUMMARY: In this series of intracerebral hematomas from aneurysmal rupture, gathered from several neurosurgical services, certain morphological features were studied in detail. Patients with very large hematomas tended to have poor neurological grades on admission to hospital and their immediate discharge outlook was correspondingly poor. Ruptured middle cerebral and pericallosal artery aneurysms were relatively common causes of in-

RÉSUMÉ: Nous avons étudié en détails certains aspects morphologiques d'une série de cas d'hématomes intra-cérébraux à la suite de rupture d'anévrysme, cas obtenus de plusieurs services neurochirurgicaux. Les patients avec de très gros hématomes ont tendance à présenter des scores neurologiques plus mauvais lors de l'admission à l'hôpital et le pronostic immédiat est également pauvre. Parmi les causes relativement fréquentes d'hématomes intracérébraux on note la rupture d'anévrysmes tracerebral hematomas. Patients with temporal lobe hematoma did relatively well; those with parietal hematoma did poorly. The larger the hematoma the less chance there was of developing cerebral vasospasm but the more likely was pre-operative brain herniation. The survival was more closely linked to size and location of the hematoma than to the location of aneurysm or the degree of midline shift.

de la cérébrale moyenne de l'artère péricalleuse. Les patients avec un hématome du lobe temporal ont un pronostic relativement bon, mais ceux avec des hématomes pariétaux évoluent plus mal. Plus l'hématome est gros, moins il y a des chances de développer des vaso-spasmes cérébraux, mais plus il $y$ $a$ de chances en faveur d'une hernie cérébrale post-opératoire. La survie est reliée beaucoup plus à la taille et à la situation de l'hématome qu'au site de l'anévrysme ou au degré de déplacement médian.
From the Departments or Divisions of Neurosurgery: Ottawa Civic Hospital, Foothills Hospital (Calgary), Vancouver General Hospital, University Hospital (London), St. Boniface Hospital (Winnipeg), Calgary General Hospital, University Hospital (Saskatoon), Hopital Notre-Dame (Montreal), Plains Health Centre (Regina), University Hospital (Edmonton), Victoria Gencral Hospital (Halifax).

Requests for Reprints to: Dr. B.K.A. Weir, 1/th Floor, Clinical Sciences Building, The University of Alberta, Edmonton, Alberta, Canada T6G 2G3.
Early knowledge of intracerebral hematomas associated with ruptured aneurysms was based principally on pathologic studies. The radiologist was on uncertain ground in attempting to differentiate clot from edema on the basis of blood vessel shifts. Now that the CT scan provides a highly accurate diagnosis, definitive surgical treatment has become a more attractive option. This report, based on pooled results from several centres attempts to correlate clinical and structural features of intracerebral hematomas from aneurysmal rupture.

\section{CLINICAL MATERIAL AND METHODS}

Data from 132 patients were obtained retrospectively from 11 medical centres. The clinical and radiological data were abstracted from the hospital chart and then transferred to computer tapes for statistical analysis.

To be included cases must have had:

1. An aneurysm demonstrated by angiography, surgery or autopsy,

2. An intracerebral hematoma ( $\mathrm{ICH})$ adjacent to the aneurysm shown on CT scan prior to surgery,

Patients may or may not have been operated; the operation may have been evacuation of the hematoma and/or clipping of the aneurysm.

Cases were graded as follows:

1. asymptomatic.

2. headache, stiff neck, drowsiness.

3. decreased consciousness with mild focal neurologic deficit.

4. decreased consciousness with major focal neurologic deficit.

5. failing vital signs, pupillary abnormalities, apparently moribund.

The size of the hematoma was measured from the CT scan using the method illustrated in Fig. 1. To allow for differences in magnification, ratios were calculated to relate the dimen- 


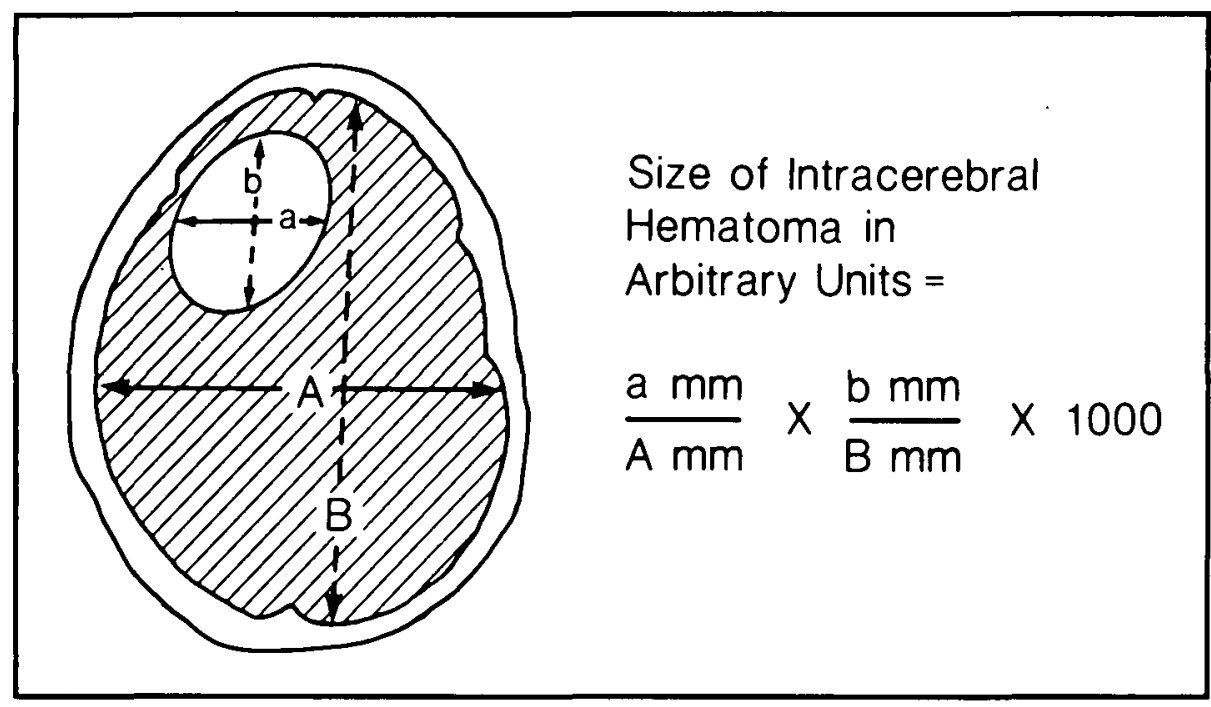

Figure 1 - The method used to quantitate the size of the intracerebral hematoma from the CT scan. This particular hematoma would have a size of 121 units and would be classified as large (between 100 and 150 units in size).

sions of the hematoma to the total dimensions of the brain in the same level. The amount of midline shift was also measured and expressed as a percentage of the width of the brain in the same plane.

\section{RESULTS}

\section{Relative importance of structural} features

A discriminant analysis of contribution to survival showed that the order or relative magnitude of structural features was size of hematoma, location of hematoma, location of aneurysm and size of midline shift.

\section{Size of hematoma}

There were significant associations (Pearson Correlation Coefficient) between the size of the hematoma and clinical grade on admission, grade at initial CT scan, grade at evacuation of hematoma and grade at clipping of aneurysm (in all $p<.001$ ) as well as grade at discharge $(\mathrm{p}<.01)$.

Similarly significant associations existed between the size of hematoma and time interval to admission, time to removal of clot and clipping of aneurysm, size of midline shift and occurence of herniation $(p<.001)$, absence of vasospasm, discharge status and survival $(\mathrm{p}<.01)$.

The mean size was $89 \pm 47$ (arbitrary units). The average size of the hematoma in 82 patients who survived was 80 (medium) while for the 50 who died it was 103 (large), the difference was significant $(p<.01$, "t-test" of comparison).

The size in 34 patients who developed vasospasm was 67 (medium). This was smaller than the mean size of 96 (medium) in 98 cases who did not get vasospasm $(p<.01)$.

The size in 54 cases who herniated pre-operatively was $113 \pm 47$ (large) while in the 78 who did not it was $72 \pm$ 39 (small) - a significant difference $(p<.001)$. The mean size of the 59 cases whose discharge grade was worse than their admission grade was $98 \pm$ 54 (medium), for the 55 cases who improved over their initial grade the size was $86 \pm 39$ (medium).

The relationship between clinical grade at initial CT scan and the size of the hematoma at that time is shown in Fig. 2. The discharge status of the patient was also assessed in relation to the size of hematoma on initial CT scan and is shown in Fig. 3.

\section{Location of hematomas}

The location of the intracerebral hematoma was as follows: frontal $46 \%$, temporal $43 \%$, parietal $10 \%$, cerebellar $1 \%$.

There were significant differences in mortality rates according to the location of the hematoma: parietal $(9 / 13=$
$69 \%)$, frontal $(24 / 60=40 \%)$, temporal $(16 / 56=29 \%)$ and cerebellar $(0 / 2=$ $0 \%),(\mathrm{p}<0.05)$.

\section{Location of aneurysm}

The artery of origin was middle cerebral in $54 \%$ of cases, anterior cerebral $25 \%$, internal carotid $15 \%$, pericallosal $5 \%$ and vertebro-basilar $1 \%$. The location of the aneurysm correlated with the location of the hematoma $(p<.01)$ and size of the hematoma $(p<.05)$. The location of the hematoma correlated with grade at discharge $(p<.05)$ and vasospasm $(\mathrm{p}<.05)$.

Mortality rate by location of aneurysm was as follows: middle cerebral $(25 / 71=35 \%)$, anterior cerebral $(16 / 33=49 \%)$, pericallosal $(1 / 7=$ $14 \%)$, internal carotid $(8 / 20=40 \%)$ and vertebro-basilar $(0 / 1=0 \%)$. Cross tabulation by Chi-square analysis of survival or death versus location did not show a significant difference.

\section{Midline shift}

The midline shift was measured in millimeters and the width of the brain at the same level, and a ratio was calculated. The overall mean midline shift was $5.2 \%$. The size of the midline shift correlated with the grade on admission, grade at initial CT scan, grade at evacuation of hematoma $(p<.001)$ and grade at clipping of aneurysm $(p<.01)$. There was also an association between midline shift and time interval to admission, CT scan, evacuation of hematoma, clipping of aneurysm, occurrence of herniation pre-operatively (all $\mathrm{p}<.001$ ).

The midline shift of 59 cases whose discharge status was worse than the admission status was $5.1 \pm 3.8 \%$; for the 55 cases who had improved by discharge it was $5.4 \pm 3.6 \%$, i.e., a greater initial midline shift. The degree of midline shift did not correlate with grade at discharge or survival.

The average midline shift was $3.8 \%$ for 34 cases who developed vasospasm and $5.6 \%$ for 97 who did not $(\mathrm{p}<.05$, "t-test" of comparison). The 53 cases who herniated pre-operatively had a midline shift of $6.5 \pm .4 \%$ which contrasts with the $4.2 \pm .3 \%$ shift of the 78 patients who did not have a pre- 


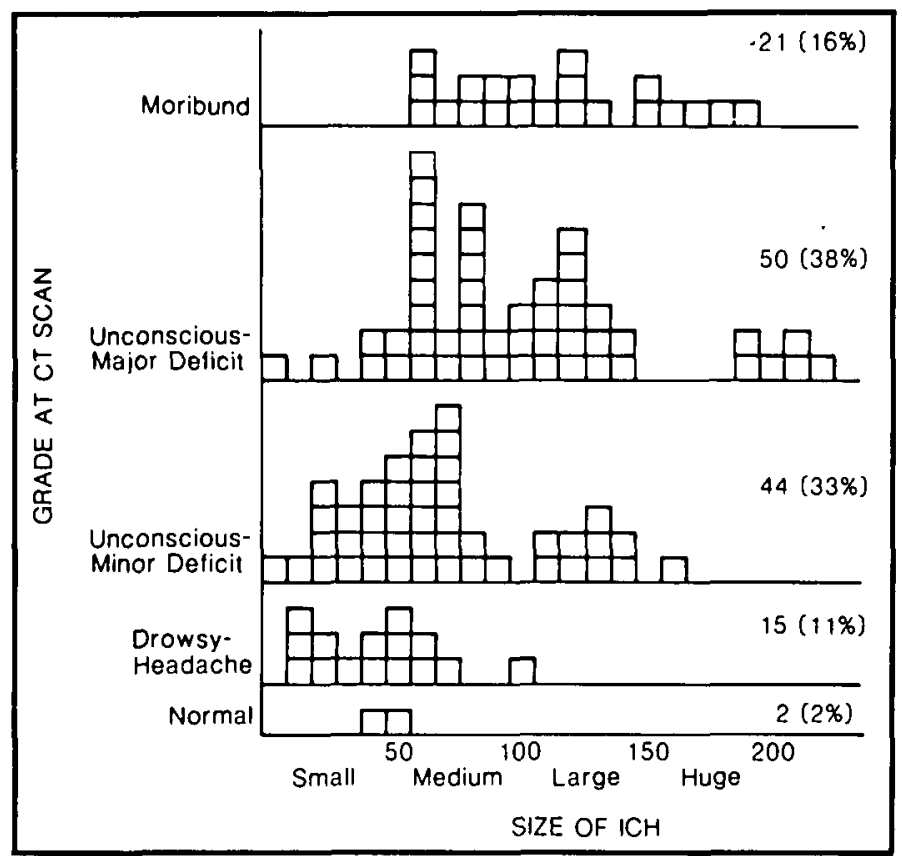

Figure 2 - Classification of cases by size of intracerebral hematoma and grade at the time of CT scan. The larger hematomas were found in patients with poor neurological grade, but there is overlap. $\mathrm{ICH}$ - intracerebral hematoma, CT - computerized tomographic.

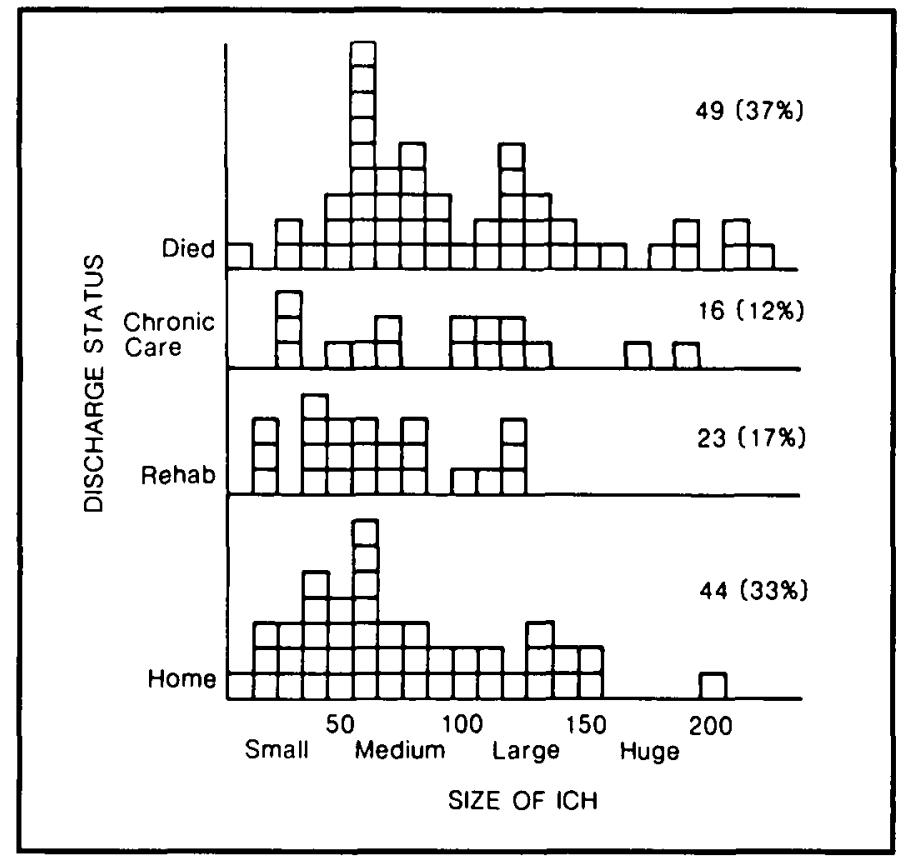

Figure 3 - Classification of cases by discharge status (destination) and size of intracerebral hematoma on initial CT scan. The presence of a large or huge hematoma was not incompatible with a return to home but it was less likely than if the hematoma was small or medium sized. ICH - intracerebral hematoma. operative herniation $(p<.01)$. The 49 who died had a midline shift of $5.5 \pm$ $.3 \%$ while the 82 survivors had a midline shift of $4.9 \pm .3 \%$. The difference was not significant.

A one-way analysis of variance failed to show significant difference in the midline shift or size of hematoma with age (Student-Neuman-Keuls procedure).

\section{DISCUSSION}

This study conclusively demonstrates that the size of the intracerebral hematoma from a ruptured aneurysm is a critical determinant of the neurological grade of the patient. The relationship between size and clinical outcome is not so tight and probably means that therapeutic removal of the hematoma can favourably affect outcome.

Steiner et al (1975) estimated the volumes of intracerebral and intraventricular hematomas in 62 cases, of which 18 were from ruptured aneurysms. They concluded that, with few exceptions, a hematoma of less than $50 \mathrm{ml}$ volume will not induce severe changes in the level of con- sciousness while one over $140 \mathrm{ml}$ will cause irreversible deterioration. Of their 4 alert aneurysm cases all had a volume under $30 \mathrm{ml} ; 1$ drowsy patient had a clot under $60 \mathrm{ml}$, and 8 comatose patients had clots between 60 and 180 $\mathrm{ml}$.

We cannot comment on the frequency with which intracerebral hematomas result from aneurysmal rupture because of the selective and retrospective manner in which our cases were obtained. The literature suggests that the answer is 30 to 40 percent of cases studied with CT scanners. An excellent correlation between CT scan and extent and location of fatal intracerebral hematomas was found by Butzer et al (1976) in 8 cases. Kendall et al (1976) in a CT study of 43 aneurysm cases found that, excluding those in fissures and ventricles, $42 \%$ had focal hematomas. Weir et al (1977) found pre-operative intracerebral hematomas on CT scans in $50 \%$ of 16 patients who died and $26 \%$ of 35 patients who survived an aneurysmal rupture. Modesti and Binet (1978) found intracerebral hematomas in $30 \%$ of 23 cases with subarachnoid hemor- rhage from aneurysm shown in CT. Bismar (1979) reported on 149 cases with acute subarachnoid hemorrhage and a clinical history suggesting aneurysmal rupture. Of 53 cerebral aneurysms having a $\mathrm{CT}$ in less than 2 days post-bleed, $40 \%$ had intracerebral hematomas. Of the 21 cases with intracerebral hematomas on early CT scans, $95 \%$ had associated subarachnoid hemorrhage. Eight cases (38\%) were from middle cerebral aneurysms, 5 (24\%) internal carotid, 4 (19\%) anterior communicating aneurysms and $4(19 \%)$ other sites. Imanaga et al (1980) studied CT scans on 168 cases of ruptured aneurysms. Within 2 weeks of hemorrhage, intracerebral hematomas were seen in $36 \%$ of anterior and middle cerebral cases, $16 \%$ of internal carotid cases and none on the posterior circulation. Cases with intracerebral hematomas showed poor neurological grade at the time of CT scan.

The generally higher incidence of intracerebral hematomas in autopsy rather than clinical or radiological series suggests that it is a risk factor for increased mortality. The incidence of 
intracerebral hematomas is 30 to 70 percent of fatal aneurysmal ruptures according to the following pathological studies.

Robertson (1947) analyzed postmortem records on 90 aneurysm cases. Seventy-two percent showed massive intracerebral hemorrhage. He found subarachnoid hemorrhage to have been more rapidly lethal than intracerebral hemorrhage. $\mathrm{He}$ suggested that intracerebral rupture was more common when aneurysms lie between cerebral surfaces, when adhesions from a previous rupture have sealed off the subarachnoid space and when aneurysms have become embedded in the brain during gradual enlargement.

Crompton (1962) studied 103 ruptured aneurysm cases at necropsy. Sixty-two $(60 \%)$ had intracerebral hematomas. Of these, $32(52 \%)$ also had cerebral infarction. Sixteen (26\%) had multiple hematomas such as both frontal lobes and cavum septum pellucidum. In 57 of 62 (92\%) of cases with intracerebral hematoma, rupture into the ventricles occurred. Twentyeight of 57 (49\%) of ventricular rupture cases had dilated ventricles. Subarachnoid hematomas ruptured into brain in $36(58 \%)$ of cases with intracerebral hematomas. They were located (in decreasing order of frequency) in the interfrontal, sylvian, olfactory, intercingulate, suprauncal and interpeduncular subarachnoid space. Direct rupture into the brain (rather than via a subarachnoid hematoma) occurred from aneurysms in the following decreasing order of frequency: internal carotid $(53 \%)$, pericallosal $(50 \%)$, anterior cerebral $(33 \%)$ and middle cerebral $(25 \%)$. Routes of rupture were as follows: anterior communicating aneurysms (i) directly via gyrus rectus or from the interfrontal space (ii) into cavum septum pellucidum then into frontal lobes; middle cerebral aneurysms (i) directly into brain, (ii) secondarily from a sylvian hematoma into external capsule then into frontal horn or trigone; pericallosal aneurysms, intercingulate subarachnoid hematoma then into frontal lobe; internal carotid-posterior communicating aneurysms, usually into temporal lobe directly; internal carotid bifurcation, into frontal lobe directly.

Freytag (1966) carried out autopsies in 250 medicolegal cases with aneurysmal rupture and unexplained sudden natural death. Sixty percent had died immediately and only $11 \%$ lived longer than 1 day. Of the 149 who died instantly, $76 \%$ had subarachnoid hemorrhage and only $24 \%$ intracerebral hemorrhage. Forty-three percent of the total had intracerebral hematomas but in only $1 \%$ of them was this the only site of hemorrhage.

Thirty-two autopsies in cases of ruptured aneurysm were reported by Tomlinson (1979). Intracerebral hemorrhage occurred in $53 \%$ and in $71 \%$ of these cases rupture into the ventricular system had occurred. Direct initial bleeding into the brain occurred in $6(35 \%)$ of hematomas.

Cancilla et al (1981) reported on the pathological examinations carried out in 206 cases from a cooperative study. Eighty-five percent died from recent aneurysmal rupture. Fifty-six percent had intracerebral and/or intraventricular or subarachnoid hemorrhage. Pure intracerebral hematoma occurred in $8 \%$.

Reynolds and Shaw (1981) reviewed the records on 205 cases dying from ruptured aneurysms. They concluded that $31 \%$ had localized hematomas potentially amenable to therapy. A history of aneurysmal rupture prior to the terminal one was obtained on 19 $(73 \%)$ of intracerebral hematoma cases. In the 41 cases having intracerebral hemorrhage and intraventricular hemorrhage, $28(68 \%)$ also gave a history of previous rupture. They collected 1548 cases of ruptured aneurysm from the literature and found that 831 (54\%) had intracerebral hemorrhage documented by pathological examination.

The present study was limited to intracerebral hematomas due to ruptured aneurysms. However, the finding of a relationship of size, location, and size of midline shift to neurological grade and outcome probably applies to hematomas of non-aneurysmal origin. McKormick and Rosenfield (1973) carried out a prospective autopsy study of 144 cases with massive nontraumatic brain hemorrhage. Known causes of the intracerebral hematomas were found in $95(66 \%)$ of cases. They were leukemia 21 (15\%), ruptured aneurysm $20(14 \%)$, angioma 15 (10\%), neoplasms 13 (9\%), nonleukemic bleeding disorders $10(7 \%)$, vasculitis $10(7 \%)$, and cortical vein thrombosis $10(7 \%)$. Of those in whom no definite cause of hemorrhage was found $37(26 \%)$ were hypertensive and 12 (8\%) were normotensive. Hypertension was defined as $\mathrm{BP}>140 / 90$ by history, or heart weight $>400$ gm if no valvular disease was present at autopsy. Ruptured aneurysm ranks closely behind hypertension and leukemia as a cause of intracerebral hematoma.

Our finding that middle cerebral and pericallosal aneurysms give rise to intracerebral hematomas relatively more frequently than other aneurysmal sites and that internal carotid and vertebrobasilar aneurysms were relatively infrequent causes, is consistent with the incidence in the pathological literature (see Table 1 and 2). It is of considerable clinical importance to know where an aneurysm is likely to be located to produce a given hematoma on a CT scan. Relevant sources are abstracted in Table 3. Our experience also was that frontal hematomas were most likely to be due to anterior cerebral aneurysms and temporal hematomas due to middle cerebral aneurysms but variations were common.

We have shown that the clinicalradiologic features of intracerebral hematomas from aneurysmal rupture are consistent with what was previously known from autopsy studies. We conclude that early evaluation of any patient with a neurological catastrophe must include a CT scan. The finding of an intracerebral hematoma should raise the strong possibility of a ruptured aneurysm being present. There is no particular type or location of hematoma which is absolutely inconsistent with an aneurysmal etiology although ganglionic and occipital sites are unusual. If angiography demonstrates a clot it should be considered that the patient is at great risk with respect to brain herniation and clinical deterioration and that this risk is proportional to its size. The extent of the midline shift 
TABLE 1

Autopsy Series: Site of Origin of Intracerebral Hematomas from Aneurysmal Rupture

\begin{tabular}{llccc} 
Artery of Origin & Robertson (1949) & Crompton (1962) & McKormick (1973) & Reynolds (1981) \\
Anterior Cerebral & $29(45 \%)$ & $27(44 \%)$ & $4(20 \%)$ & $46(46 \%)$ \\
Middle Cerebral & $24(37 \%)$ & $16(26 \%)$ & $11(55 \%)$ & $33(33 \%)$ \\
Pericallosal & $6(9 \%)$ & $6(10 \%)$ & $0(0 \%)$ & (included with \\
& & & & anterior cerebral) \\
Internal carotid & $4(6 \%)$ & $13(21 \%)$ & $4(20 \%)$ & $13(13 \%)$ \\
Vertebro-basilar & $2(3 \%)$ & $0(0 \%)$ & $1(5 \%)$ & $8(8 \%)$ \\
Total Cases & $65(100 \%)$ & $62(100 \%)$ & $20(100 \%)$ & $100(100 \%)$ \\
\hline
\end{tabular}

TABLE 2

Autopsy Series: Frequency of Aneurysmal Rupture Producing Intracerebral Hematoma

$\begin{array}{lccc}\text { Site of Rupture } & \text { Robertson (1949) } & \text { Crompton (1962) } & \text { Freytag (1966) } \\ \text { Pericallosal } & 6 / 7(86 \%) & 6 / 6(100 \%) & 15 / 22(68 \%) \\ \text { Anterior Cerebral } & 29 / 34(85 \%) & 27 / 35(77 \%) & 25 / 59(42 \%) \\ \text { Middle Cerebral } & 24 / 29(83 \%) & 16 / 34(47 \%) & 45 / 68(66 \%) \\ \text { Internal Carotid } & 4 / 9(44 \%) & 13 / 27(48 \%) & 18 / 62(29 \%) \\ \text { Vertebro-basilar } & 2 / 8(25 \%) & 0 / 0(0 \%) & 4 / 35(11 \%) \\ \text { Total } & 65 / 87(75 \%) & 62 / 102(61 \%) & 107 / 246(44 \%)\end{array}$

Site of Hematoma

Frontal

Author

(Crompton 1962)

(Robertson 1947)

Temporal

(Crompton 1962)

(Robertson 1947)

Parietal
(Crompton 1962)

(Robertson 1947)
TABLE 3

\section{Artery of Origin}

Anterior Cerebral (69\%) Pericallosal (13\%) Middle Cerebral (9\%)

Internal Carotid (9\%)

Anterior Cerebral (72\%) Middle Cerebral (14\%) Pericallosal (10\%)

Internal Carotid (3\%)

Middle Cerebral (50\%) Internal Carotid (50\%)

Middle Cerebral (86\%) Internal Carotid (14\%)

Anterior Cerebral (67\%) Middle Cerebral (33\%)

Middle Cerebral (100\%) is a less sensitive indicator overall because it does not reflect temporal lobe masses initially. A patient with an intracerebral hematoma is less likely to do well than a patient who has a pure subarachnoid hemorrhage. The patient who will do worst is the one with a huge parietal clot. The best recoveries occur, at least in the short term, with temporal lobe hematomas. The "protective" effect of an intracerebral hematoma with respect to development of vasospasm may reflect the fact that if all the bleeding is into the brain substance there is a smaller amount of blood in the subarachnoid space bathing the arteries with spasmogens. Early evacuation of the clot permits clipping of the aneurysm so that the twin danger of herniation from the mass and rebleeding are obviated.

Our combined data has permitted further firm correlations between the size and location of intracerebral hematomas and other clinical and radiological features found in the patient with a ruptured aneurysm.

\section{ACKNOWLEDGEMENTS}

We appreciate the financial support of Ericka and Henry Schroeder and the assistance of Michael Grace, Ph.D. in statistical analysis of the data.

\section{REFERENCES}

BISMAR, J. (1979): Computer tomography as the primary radiologic procedure in acute subarachnoid hemorrhage. Acta Radiologica (Diagnosis), 20: 849-864.
BUTZER, J.F., CANCILLA, P.A., CORNELL, S.H. (1976): Computerized axial tomography of intracerebral hematoma. Archives of Neurology, 33: 206-214.

CANCILLA, P.A., TORNER, J.C., NIBBELINCK, D.W. (1981): Pathologic results from patients who died in randomized study (Phase II). In Sahs, A.L., Nibbelinck, D.W., Torner, J.C., Eds. Aneurysmal Subarachnoid Hemorrhage. Urban \& Schwarzenberg, Baltimore - Munich, 369 pp.

CROMPTON, M.R. (1962): Intracerebral hematoma complicating ruptured cerebral berry aneurysm. Journal of Neurology, Neurosurgery and Psychiatry, 25: 378-386.

FREYTAG, E. (1966): Fatal rupture of intracranial aneurysms. A survey of 250 medicolegal cases. Archives of Pathology, 81: 418-424. 
IMANAGA, H., YAMAMOTO, M., JIMBO, M., KITAMURA, K., KOBAYASHI, N., SAITO, Y. (1980): Computed tomography in the diagnosis of hemorrhage secondary to intracranial aneurysm. Neurological Surgery (Tokyo), 8: 623-631 (Japanese).

KENDALL, B.E., LEE, B.C., CLAVERIA, E. (1976): Computerized tomography and angiography in subarachnoid hemorrhage. British Journal of Radiology, 49: 483-501.

McCORMICK, W.F,, ROSENFIELD, D.B. (1973): Massive brain hemorrhage: $A$ review of 144 cases and an examination of their causes. Stroke, 4: 946-954.
MODESTI, L.M., BINET, E.F. (1978): Value of computed tomography in the diagnosis and management of subarachnoid hemorrhage. Neurosurgery, 3: 151-156.

REYNOLDS, A.F., SHAW, C.M. (1981): Bleeding patterns from ruptured intracranial aneurysms: An autopsy series of 205 patients. Surgical Neurology, 15: 232-235.

ROBERTSON, E.F. (1949): Cerebral lesions due to intracranial aneurysms. Brain, 72 : 150-185.
STEINER, L., BERGVALL, U., ZWETNOW, N. (1975). Quantitative estimation of intracerebral and intraventricular hematoma by computer tomography. Acta Radiologica Supplement, 346: 143-154.

TOMLINSON, B.E. (1959): Brain changes in ruptured intracranial aneurysm. Journal of Clinical Pathology, 12: 391-399.

WEIR, B., MILLER, J., RUSSELL, D. (1977): Intracranial aneurysms: A clinical, angiographic and computerized tomographic study. Canadian Journal of Neurological Sciences, 4: 99-105. 